\title{
MASA QUÍSTICA RETROPERITONEAL GIGANTE: MUCOCELE APENDICULAR
}

\author{
A. RODRÍGUEZ ALONSO, G. SUÁREZ PASCUAL, C. BONELLI MARTÍN, \\ A. GONZÁlEZ BLANCO, J. LORENZO FRANCO, M.Á. CUERPO PÉREZ, \\ C. NOGUEIRA CARBALLEDO*, J.C. ÁlVAREZ FERNÁNDEZ*, J. NIETO GARCÍA
}

Servicio de Urología. *Servicio de Anatomía Patológica. Hospital Arquitecto Marcide. El Ferrol (La Coruña).

Actas Urol Esp. 28 (4): 327-331, 2004

\section{RESUMEN}

MASA QUÍSTICA RETROPERITONEAL GIGANTE: MUCOCELE APENDICULAR

Mucocele apendicular es un término que define la dilatación del apéndice vermiforme producida por acumulación intraluminal de moco. Son cuatro los procesos patológicos descritos que pueden ocasionar un mucocele apendicular: obturación de la comunicación cecoapendicular, hiperplasia mucosa, cistoadenoma mucinoso y cistoadenocarcinoma mucinoso. De todos ellos, el cistoadenoma mucinoso es el más frecuente, observándose en el 50\% de los mucoceles apendiculares.

El 6\% de los pacientes con mucocele apendicular desarrollan pseudomixoma peritoneal, posiblemente por diseminación de células epiteliales al interior de la cavidad abdominal.

Es de gran importancia el diagnóstico preoperatorio de la lesión, para manipularla cuidadosamente durante el acto quirúrgico. El TAC es la exploración radiológica más precisa, aunque no existen signos patognomónicos de mucocele. En el TAC el mucocele se presenta como una masa quística bien delimitada, que comprime el ciego, sin reacción inflamatoria periférica, y con bajos niveles de atenuación, que oscilan entre densidad agua y tejido blando.

Presentamos un caso de mucocele apendicular ocasionado por un cistoadenoma mucinoso, que se presentó clínicamente como masa retroperitoneal gigante. El diagnóstico fue efectuado post-operatoriamente, tras el estudio anatomopatológico de la pieza quirúrgica.

PALABRAS CLAVE: Mucocele. Apéndice. Cistoadenoma mucinoso. Retroperitoneo.

\section{ABSTRACT}

GIANT RETROPERITONEAL CYSTIC MASS: APPENDICEAL MUCOCELE

Appendiceal mucocele is a term used to describe the dilatation of the vermiform appendix produced by an intraluminal accumulation of mucus. Four pathological processes have been described that may lead to an appendiceal mucocele: obturation of cecoappendiceal communication, mucosal hyperplasia, mucinous cystadenoma and mucinous cystoadenocarcinoma. The most frequent is mucinous cystadenoma, seen in $50 \%$ of appendiceal mucoceles.

$6 \%$ of patients with appendiceal mucocele develop peritoneal pseudomixoma, possibly through dissemination of the epithelial cells into the abdominal cavity.

Preoperative diagnosis of the lesion is particularly important in order to deal with it carefully during surgery. CT scan is the most precise radiological exploration, although there are no pathognomonic signs of mucocele. Typical CT finding of a mucocele is a well-defined cystic mass that compresses the cecum without any peripheral inflammatory reaction, and with low levels of attenuation that vary between water and soft tissue density.

We present a case of an appendiceal mucocele caused by a mucinous cystadenoma clinically presented as a giant retroperitoneal mass. Diagnosis was postoperatively made, after pathological study of the surgical sample.

KEY WORDS: Mucocele. Appendix. Mucinous cystoadenoma. Retroperitoneum. 
$\mathrm{M}$ ucocele apendicular es un término inicialmente descrito por Rokitansky en $1842^{1}$, que define una dilatación del apéndice vermiforme producida por acumulación intraluminal de moco $^{2}$. Se trata de una entidad clínica que puede estar ocasionada por cuatro procesos patológicos subyacentes diferentes:

1. Obturación de la comunicación cecoapendicular por un fecalito o retracción cicatricial.

2. Hiperplasia mucosa focal o difusa, sin atipias celulares.

3. Cistoadenoma mucinoso, con cierto grado de atipia celular.

4. Cistoadenocarcinoma mucinoso ${ }^{1-3}$.

La diseminación de células epiteliales del mucocele al interior de la cavidad abdominal ocurre en el 6\% de los casos y se denomina pseudomixoma peritoneal ${ }^{4}$.

Presentamos un caso de mucocele apendicular que se presentó clínicamente como masa retroperitoneal gigante, y cuyo diagnóstico fue efectuado post-operatoriamente, tras el estudio anatomopatológico de la pieza quirúrgica.

\section{CASO CLÍNICO}

Varón de 66 años remitido por el Servicio de Gastroenterología ante el hallazgo de masa retroperitoneal de $25 \mathrm{~cm}$, en ecografía abdominal realizada para estudio de dispepsia. Entre sus antecedentes personales destacaban: tuberculosis pulmonar antigua y enfermedad pulmonar obstructiva crónica. En la inspección física se observaba una gran masa que deformaba hemiabdomen anterior derecho, extendiéndose desde el área subcostal hasta el pubis. A la palpación, dicha masa era indolora, de consistencia firme, sin signos de irritación peritoneal y con matidez a la percusión. Al tacto rectal se palpaba una próstata de tamaño II/V, adenomatosa, lisa y bien delimitada. La analítica sanguínea, el sedimento y el cultivo de orina, eran normales, y la cifra de PSA de 1,2 ng/ml.

La ecografía abdominal mostraba una lesión quística de $25 \mathrm{~cm}$ de diámetro, con abundantes ecos internos, que se extendía desde el borde inferior del hígado hasta la ingle.

En la urografía i.v. (UIV) se observaba distorsión de la silueta y la pelvis renales derechas, con un importante desplazamiento del segmento lumbo-iliaco del uréter derecho, que sobrepasaba la línea media abdominal, así como ligera ectasia del tracto urinario superior ipsilateral (Fig. 1).

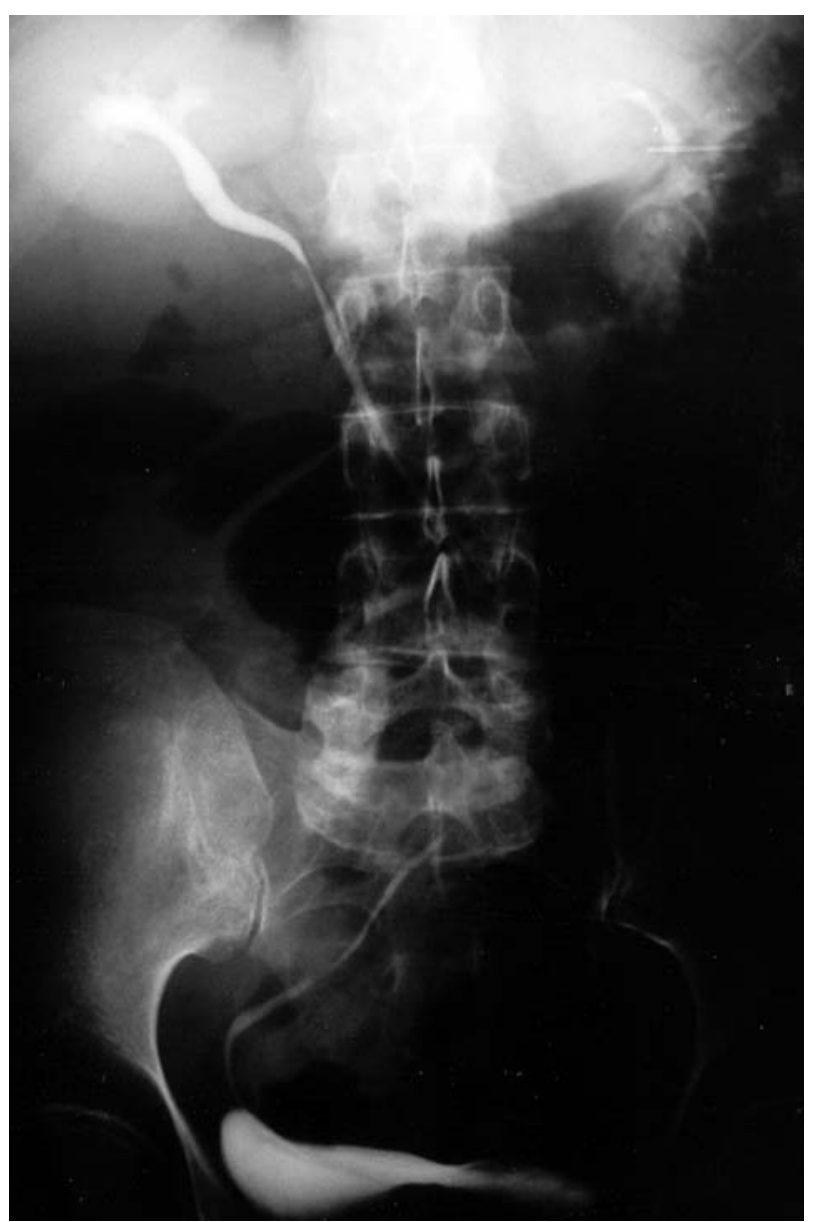

FIGURA 1. UIV que muestra una distorsión de la silueta renal derecha, asi como un importante desplazamiento medial del uréter lumbo-iliaco derecho.

El TAC abdomino-pélvico realizado con contraste oral e intravenoso, ponía de manifiesto una masa quística polilobulada retroperitoneal derecha de 25 cm de diámetro cráneo-caudal, que se extendía desde la región subhepática hasta la ingle, comprimiendo y desplazando el riñón derecho, el músculo psoas ilíaco y el colon ascendente, en sentido posterior y medial. Se observaban asimismo calcificaciones puntiformes en la pared quística (Figs. 2 y 3). En la porción medial e inferior de dicha masa se evidenciaba una estructura tubular de $2 \mathrm{~cm}$ de diámetro y $7 \mathrm{~cm}$ de longitud, con afilamiento progresivo en sentido caudal, finalizando en stop completo. El riñón derecho se mostraba funcionalmente normal. Los hallazgos del TAC eran interpretados como un posible hemirriñón inferior derecho displásico con agenesia parcial del uréter.

Ante las dudas diagnósticas existentes con los estudios radiológicos efectuados, se decidía 


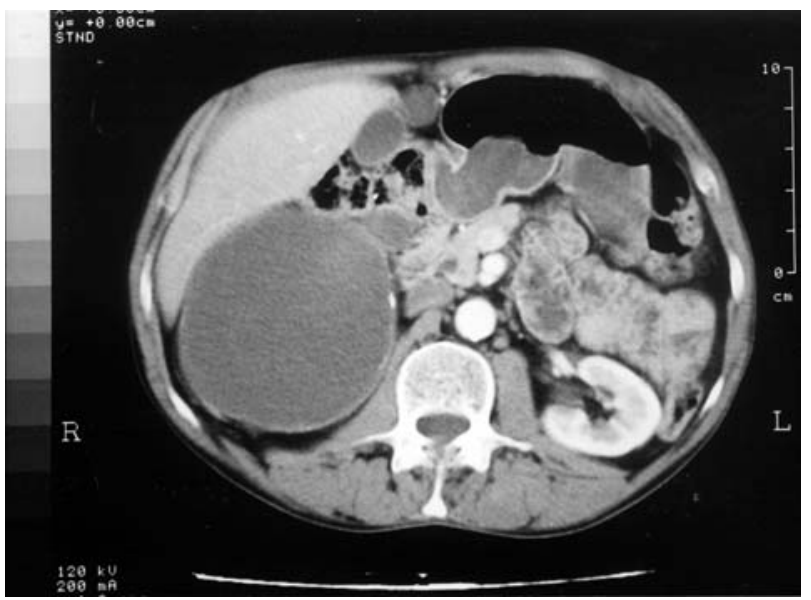

FIGURA 2. TAC abdominal en el que se observa una gran masa quística homogénea retroperitoneal con calcifica ciones en su pared, que desplaza medialmente el ángulo hepático del colon.



FIGURA 3. Detalle de TAC abdominal en el que se aprecia un área de la masa de morfología polilobulada, con tabiques finos y calcificaciones parietales puntiformes.

efectuar una punción-biopsia percutánea, que era informada como pared de lesión quística, y citología de orina, que no evidenciaba células malignas.

Posteriormente se realizaba intervención quirúrgica mediante abordaje pararrectal derecho, observando una masa quística relacionada en su extremo craneal con el lóbulo hepático derecho y el polo inferior del riñón, y en su extremo caudal con el espacio de Retzius y el orificio inguinal interno. No se observaba infiltración de ningún órgano intra-abdominal. Se efectuó resección cuidadosa de la masa quística y del apéndice cecal, que se hallaba en íntima relación con la porción caudal de la misma (Fig. 4).

En el estudio anatomopatológico de la pieza se apreciaba un apéndice dilatado, revestido por un epitelio mucinoso citológicamente benigno, que formaba estructuras de tipo papilar (Fig. 5).

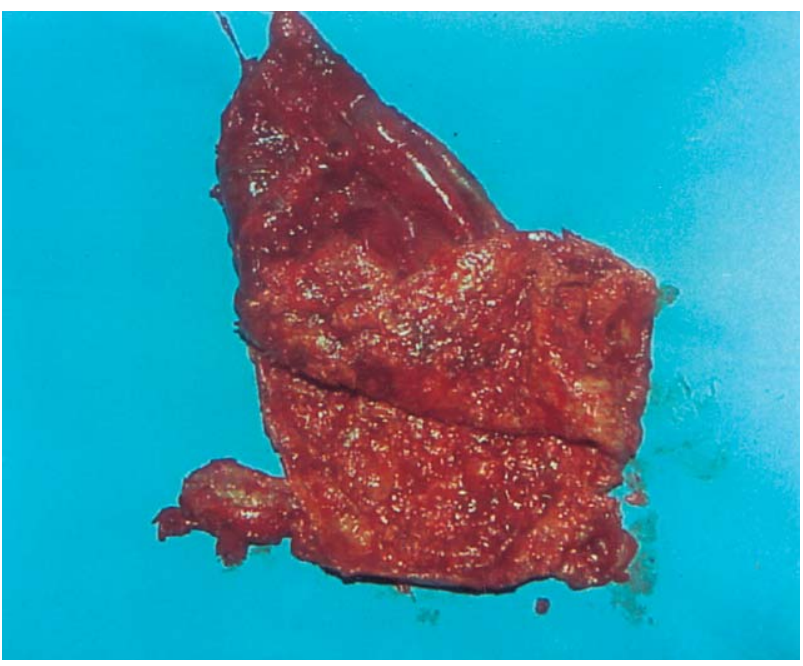

FIGURA 4. Pieza de mucocelectomía tras el vaciado de su contenido.

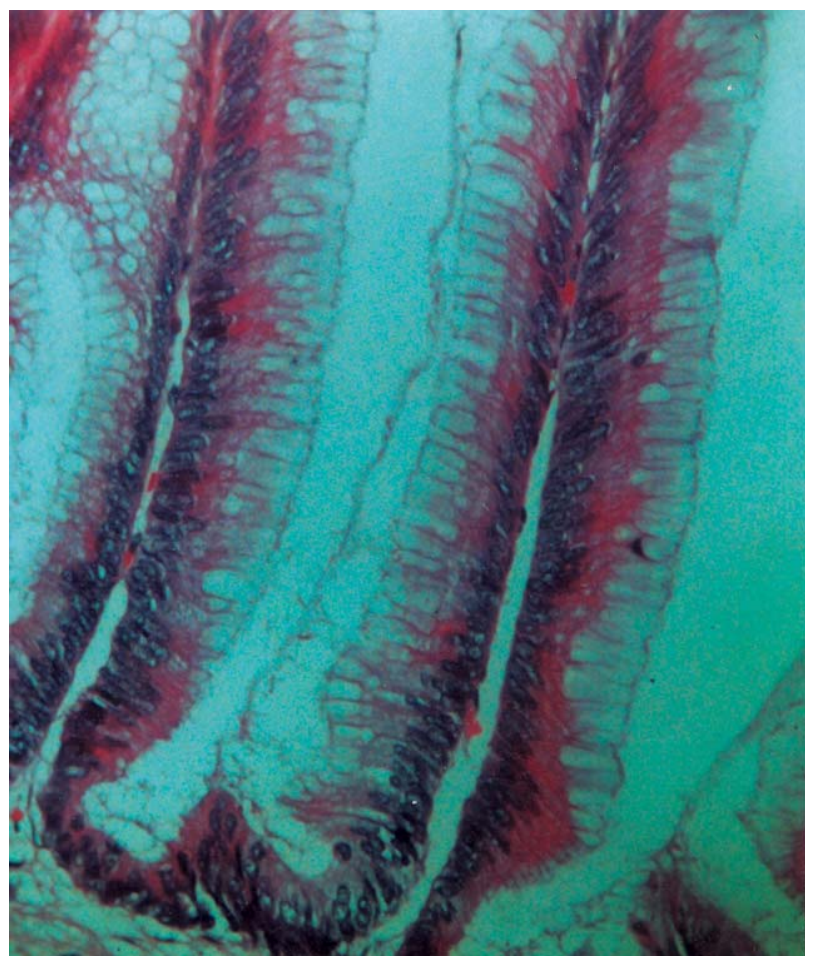

FIGURA 5. Fotografia a $40 X$ en la que se observa una luz apendicular dilatada, revestida por epitelio mucinoso cito lógicamente benigno, que forma estructuras de tipo papilar. 
Dichos hallazgos resultaban diagnósticos de cistoadenoma mucinoso de apéndice.

El post-operatorio cursaba con normalidad. En la UIV efectuada a los tres meses de la intervención quirúrgica se observaba una buena función renal bilateral, con hipercorrección lateral del trayecto ureteral derecho y desaparición de la distorsión renal derecha (Fig. 6).

En la revisión efectuada a los 20 meses, el paciente se encuentra asintomático desde el punto de vista urológico, y en el TAC de control no se evidencian lesiones abdominales sugestivas de pseudomixoma peritoneal.

\section{DISCUSIÓN}

El cistoadenoma mucinoso es la causa más frecuente de mucocele apendicular, representando el $50 \%$ de los casos 3 .

Desde el punto de vista epidemiológico, la incidencia de mucocele aumenta a partir de la sexta década de la vida ${ }^{1,3,5}$, observándose en el $0,2-0,7 \%$ de las piezas de apendicectomía ${ }^{2,3,6}$ y en el $0,15 \%$ de las necropsias ${ }^{6,7}$.

El mucocele se asocia a neoplasias de otros órganos intra-abdominales, como el adenocarcinoma de colon $(0-21 \%)$ y ovario $(4-24 \%)^{2,4}$. Las pacientes con cistoadenocarcinoma ovárico y metástasis peritoneales, presentan casi invariablemente una neoplasia mucinosa apendicular asociada, sincrónica o metacrónica. Es por ello que algunos autores se cuestionan si se trata de neoplasias primarias independientes, o bien la neoplasia de ovario es secundaria a implantes de la neoplasia apendicular ${ }^{2,4}$.

La sintomatología del mucocele es inespecífica, presentándose como hallazgo incidental durante una intervención quirúrgica, una exploración radiológica o un procedimiento endoscópico, en el 23-50\% de los $\operatorname{casos}^{2,4}$.

La presentación clínica más frecuente es el dolor hemiabdominal derecho, agudo o crónico, y la presencia de masa abdominal palpable, como ocurría en el paciente de nuestro caso $^{1,2}$. Con menos frecuencia se observan signos y síntomas derivados de la afectación del aparato digestivo (rectorragia, oclusión intestinal, abdomen agudo) y del aparato urinario (cólico nefrítico, insuficiencia renal obstructiva, hematuria) ${ }^{2,7}$. La presentación en forma de dolor abdominal progresivo y

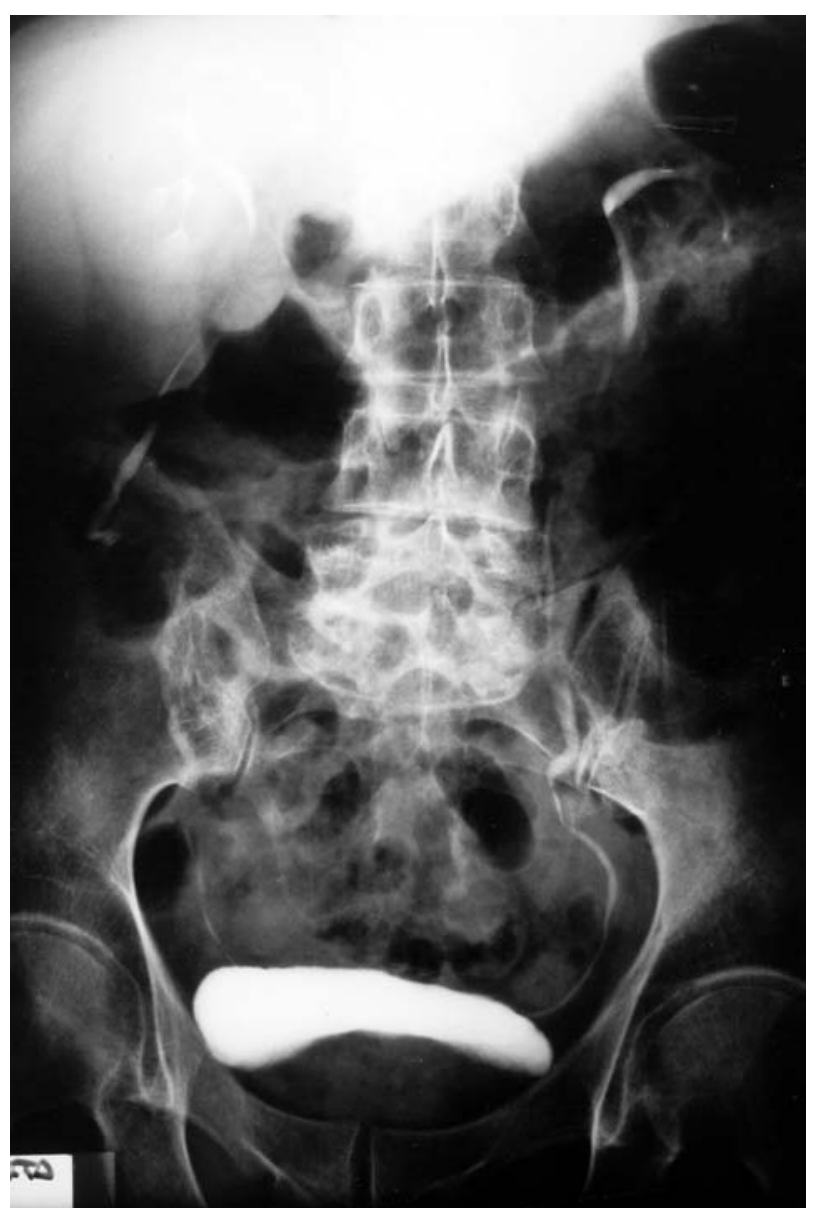

FIGURA 6. UIV post-operatoria en la que se observa una hipercorrección lateral del trayecto ureteral derecho.

sensación de plenitud es característica de pseudomixoma peritoneal ${ }^{4}$.

Los hallazgos de laboratorio son inespecíficos, aunque en los mucoceles ocasionados por cistoadenocarcinoma pueden detectarse niveles elevados de antígeno carcinoembrionario ${ }^{2}$.

La ecografía y el enema baritado se han empleado en el diagnóstico radiológico de mucocele. Mediante ecografia puede observarse una lesión quística encapsulada, con una ecogenicidad dependiente de la cantidad de mucina ${ }^{1,5}$. En el enema opaco, la existencia de pliegues cecales de morfología espiral, o bien un defecto de repleción apendicular, son hallazgos compatibles con mucocele apendicular ${ }^{1}$.

Probablemente el TAC es la técnica más precisa en el diagnóstico radiológico de mucocele apendicular. En el TAC el mucocele se presenta como una masa quística bien delimitada, que comprime el ciego, sin reacción inflamatoria periférica, con 
bajos niveles de atenuación, que oscilan entre densidad agua y tejido blando. Con cierta frecuencia se observan calcificaciones parietales puntiformes o curvilíneas ${ }^{1,4,5}$. La existencia de ascitis o de masas de densidad tejido blando, comprimiendo vísceras abdominales, sin infiltrarlas, sugiere pseudomixoma peritoneal ${ }^{4,6}$. El paciente de nuestro caso presenta una gran masa quística, con abundantes calcificaciones murales puntiformes y sin datos de implantación peritoneal, como se puede observar en las Figuras 3 y 4.

La reconstrucción coronal multiplanar mediante TAC puede resultar útil en la planificación de la intervención quirúrgica, ya que muestra de forma precisa las relaciones anatómicas de la lesión ${ }^{5}$.

Los hallazgos del TAC no son totalmente específicos de mucocele, por lo que debe realizarse diagnóstico diferencial con quiste mesentérico, duplicación intestinal y linfocele. En pacientes femeninas deben considerarse asimismo el quiste ovárico derecho y el hidrosalpinx ${ }^{4,6}$.

Es de gran importancia el diagnóstico preoperatorio, para manipular cuidadosamente la lesión durante la intervención quirúrgica, evitando la rotura de la misma, por la posibilidad de siembra peritoneal de células malignas ${ }^{1,5}$. Para efectuar el diagnóstico preoperatoriamente es necesario tener un alto índice de sospecha, ya que no existe ningún dato clínico, ni radiológico, patognomónico de mucocele. En la mayoría de los casos el diagnóstico se realiza post-operatoriamente, mediante el estudio anatomopatológico ${ }^{8}$.

El tratamiento de elección del cistoadenoma mucinoso de apéndice es la exéresis cuidadosa de la lesión ${ }^{2,3}$. En ocasiones puede ser preciso ampliar la resección al segmento de ciego o íleon que se halle afectado ${ }^{4,8}$. El abordaje laparoscópico es discutido, por el riesgo de ruptura de la lesión 6 .

Si existe pseudomixoma peritoneal, causado por una perforación espontánea o yatrógena del mucocele, es preciso realizar una revisión minuciosa de la cavidad abdominal, efectuando exéresis de cualquier implante mucinoso ${ }^{3}$.
La supervivencia del cistoadenoma mucinoso de apéndice es del 91-100\% a los 5 años de seguimiento, independientemente de la perforación o no de la lesión. En el lado opuesto, el cistoadenocarcinoma asociado a pseudomixoma peritoneal presenta una supervivencia del $25 \%$ a los 5 años $^{3,4}$.

\section{REFERENCIAS}

1. MINNI F, PETRELLA M, MORGANTI A, SANTINI D, MARRANO D.: Giant mucocele of the appendix. Report of a case. Dis Colon Rectum 2001; 44: 10341036.

2. HARITOPOULOS KN, BROWN DC, LEWIS P, MANSOUR F, ELTAYAR AR, LABRUZZO $\mathrm{C}$ et al.: Appendiceal mucocoele: a case report and review of the literature. Int Surg 2001; 86: 259-262.

3. DE PABLO CÁRDENAS A, LOZANO URUÑUELA F, PINÓS PAUL MA, JIMÉNEZ ARISTU JI, JIMÉNEZ CALVO JM, RUIZ RAMO M et al.: Obstrucción ureteral extrínseca secundaria a mucocele apendicular. Arch Esp Urol 2001; 54 (5): 451-454.

4. ZISSIN R, GAYER G, KOTS E, APTER S, PERI M, SHAPIRO-FEINBERG M.: Imaging of mucocele of the appendix with emphasis on the CT findings: a report of 10 cases. Clin Radiol 1999; 54: 826-832.

5. WALLIS JW, MADAN A, SHOEMAKER MC.: Giant mucocele of the appendix. AJR 1995; 165: 10131014.

6. PEREIRA ARIAS JG, IBARLUZEA GONZÁLEZ JG, GUTIÉRREZ DÍEZ JM, MURUETA-GOYENA MENDIZÁBAL J, ÁLVAREZ MARTÍNEZ A.: Lesión quística retrovesical inusual: mucocele apendicular. Arch Esp Urol 2002; 55 (5): 560-564.

7. PARADA R, ROSALES A, ALGABA F, LLUIS F, VILLAVICENCIO H.: Mucocele of the appendix: an unusual cause of obstructive kidney failure. BJU Int 1998; 82: $444-445$.

8. SATO T, UEDA T, KON H, YAGIHASHI S, KOTANAGI H.: Mucinous cystadenoma of appendix concomitant with perityphlic granuloma. J Gastroenterol 1995; 30: $779-782$

\footnotetext{
Dr. A. Rodríguez Alonso

Servicio de Urología

Hospital Arquitecto Marcide

Ctra. San Pedro de Leixa, s/n

15405 El Ferrol (La Coruña)
}

(Trabajo recibido el 4 julio de 2003) 